\title{
Philosophiques
}

\section{Binkelmann, Christoph, Theorie der praktischen Freiheit. Fichte - Hegel, Berlin, De Gruyter, 2007, 376 p.}

\section{Quentin Landenne}

Volume 36, numéro 1, printemps 2009

L’idéalisme britannique

URI : https://id.erudit.org/iderudit/038024ar

DOI : https://doi.org/10.7202/038024ar

Aller au sommaire du numéro

Éditeur(s)

Société de philosophie du Québec

ISSN

0316-2923 (imprimé)

1492-1391 (numérique)

Découvrir la revue

Citer ce compte rendu

Landenne, Q. (2009). Compte rendu de [Binkelmann, Christoph, Theorie der praktischen Freiheit. Fichte - Hegel, Berlin, De Gruyter, 2007, 376 p.]

Philosophiques, 36(1), 253-256. https://doi.org/10.7202/038024ar d'utilisation que vous pouvez consulter en ligne. 
ici s'insère difficilement dans la recherche la plus récente sur ces sujets, même s'il ne faut pas oublier qu'il vise d'abord et avant tout un public francophone. Ces réserves ne doivent pas masquer l'intérêt réel de ce livre utile et pertinent pour quiconque tâcherait de mettre à jour les fondements conceptuels et historiques du libéralisme. Une telle entreprise mérite d'être saluée.

CHRISTIAN NADEAU

Université de Montréal

\section{Binkelmann, Christoph, Theorie der praktischen Freiheit. Fichte - Hegel, Berlin, De Gruyter, 2007, 376 p.}

Le livre de C. Binkelmann se propose d'analyser un des projets les plus centraux de l'idéalisme allemand, l'édification scientifique d'un système de la liberté entendu comme système de la nécessité interne de la raison qui pose la liberté comme principe constitutif du monde. Ce projet d'une théorie de la liberté pratique, l'A. l'étudie à partir de deux de ses versions les plus fortes et les plus profondes, en confrontant principalement la première philosophie de J. G. Fichte au système plus tardif de G. W. Hegel. Cette confrontation part de l'hypothèse que l'influence du premier sur le second est à ce point prégnante qu'il est possible de considérer la philosophie pratique de Hegel comme une réplique aux positions de Fichte.

L'A. y défend la thèse que ces deux systèmes construisent leur concept de liberté pratique sous deux aspects sinon contradictoires, du moins radicalement divergents dans leur principe respectif. Alors que le système fichtéen insiste sur le caractère absolu d'une liberté infondée par quoi que ce soit d'autre qu'elle-même (Grundlosigkeit) et développe son concept de liberté pratique comme origine (Ursprung) et saut discontinu (Sprung), le système hégélien produit une fondation absolue de la liberté et constitue même l'exposition de sa genèse rationnelle. L'origine et la genèse sont ainsi identifiées comme les deux pôles ou les deux points de vue systématiques possibles de ce système de la liberté.

Le livre se compose de trois parties: les deux premières exposent successivement une interprétation interne des perspectives fichtéenne et hégélienne, en partant de l'ancrage systématique fondamental du concept de liberté pratique pour aboutir aux domaines plus concrets d'effectuation de ce concept chez ces deux philosophes; dans une troisième partie, l'A. cherche à construire méthodologiquement et à investir philosophiquement une position plus externe et critique lui permettant de confronter directement les deux systèmes l'un à l'autre et de révéler à partir de leurs oppositions ce qu'il y aurait d'unilatéral en chacun d'eux.

Le premier pas vers un système de la liberté réside dans la liberté de la réflexion et du choix d'un système. Le philosophe est placé devant l'alternative entre le moi en soi de l'idéalisme et la chose en soi du réalisme (dogmatisme); ce conflit d'intérêts originaire entre des points de vue irréductiblement opposés et empêchant toute critique interne ne peut être résolu que par un moment décisionniste. Seule la décision idéaliste, qui repose sur un intérêt pratique, une croyance en la liberté, peut se confirmer par une autojustification systématique réflexivement cohérente, qui ne supprime pour autant jamais le moment pratique ou décisionniste originaire. 
Cette décision ouvre une «chaîne idéaliste d'implications » qui mène au point de vue transcendantal: 1) la conscience d'un objet X suppose 2) la conscience de la conscience de cet objet X, qui suppose 3) la conscience de la conscience en général, la conscience de soi. Par cette conscience de soi ou cette intuition intellectuelle, Fichte n'entend pas l'intuition suprasensible d'une chose en soi à laquelle Kant avait opposé l'interdit de la philosophie critique, mais "l'intuition de l'auto-activité et de la liberté » par laquelle Fichte cherche à combler le déficit de l'idéalisme kantien. En effet, le point de vue transcendantal de l'intuition intellectuelle vient compléter et fonder l'unité des instances cognitives constitutives de la conscience empirique, soit l'intuition sensible et le concept de l'objet représenté. Cette intuition intellectuelle, ou Tathandlung (littéralement "action-fait»), doit être comprise sous un double aspect: d'une part, en tant que forme abstraite de l'auto-position, elle est " une simple abstraction, une simple pensée du philosophe", qui n'apparaît jamais comme un objet de la conscience concrète, précisément parce qu'elle est le fondement qui rend cette conscience possible. Mais, d'autre part, l'intuition intellectuelle doit aussi être comprise comme intuition réelle et concrète opérant en chaque acte de la conscience empirique, comme un mode de connaissance permanent sans lequel je "ne peux faire le moindre pas, bouger la main ou le pied» (p. 20-27).

Cette conception de la liberté comme origine absolue et infondée implique une discontinuité de principe entre les points de vue transcendantal et empirique, entre philosophie et vie. La liberté procède originairement d'un intérêt pratique, d'une croyance fondamentale en la liberté. La philosophie part du principe de cette croyance, saisie transcendentalement dans l'intuition intellectuelle, pour en déduire les structures de la conscience; mais cette croyance, qui est en même temps l'origine absolue de la liberté humaine, la philosophie ne peut la fonder ni la prouver davantage, car elle ne peut la transcender. L'effort pratique qui définit la conscience et l'action humaines répond à la croyance en la liberté et la réalise infiniment dans une tension entre infini et fini, moi et non-moi. Cette tension ne peut être totalement dissoute ni dans le système philosophique ni dans la vie de la liberté tant elle constitue essentiellement l'auto-compréhension de l'humanité. Le philosophe ne peut donc résoudre systématiquement ou philosophiquement l'opposition entre philosophie et vie, mais il peut contribuer à leur unité morale dans la vie, en travaillant pour son époque au devenir transparent à elle-même de la croyance en la liberté. Il s'ensuit de cette finalité éthique du sujet libre un primat de la constitution de sens (Sinnstiftung) subjective sur la réalité objective: le monde devient le «corps» de ce sujet, et les domaines objectifs de la coexistence humaine (la société, le droit, l'État, la culture) tirent leur sens en tant que moyens de cette finalité morale (p. 146; 333-341).

La critique de Fichte par Hegel coïncide avec les débuts de son propre système et sera renforcée par le développement ultérieur de ce système. Du point de vue hégélien - que la deuxième partie de l'ouvrage se consacre à expliciter pour lui-même, avant de le confronter à nouveau au point de vue fichtéen dans la troisième partie - la philosophie fichtéenne représente l'accomplissement du transcendantalisme critique, non son dépassement: elle systématise le point de vue transcendantal, qui est un point de vue artificiel sur la vie, et non un point de vue de la vie sur elle-même. Elle est une critique de la connaissance, une réflexion de la réflexion. Le principe de l'identité sujet-objet dont elle part reste unilatéralement subjectif, car il procède de l'abstraction de la conscience pure à partir de la conscience empirique qui est caractérisée par l'opposition sujet-objet. Par cette abstraction, le point de vue transcendantal 
reste dépendant de cette opposition, qu'il perpétue dans son opposition au point de vue empirique de la conscience. La philosophie transcendantale ne peut ramener à son principe unitaire la conscience empirique et factuelle qu'elle présuppose que par une déduction sans fin de cette conscience qui n'aboutit jamais au système accompli de l'identité absolue des deux consciences opposées. Les principes de la Doctrine de la science de Fichte n'exposent pas l'auto-construction de l'absolu dans et pour le savoir absolu, mais relativisent l'absolu dans la simple forme de la réflexion. Les deux premiers principes, l'auto-position du moi et l'opposition du moi au non-moi sont réciproquement dépendants, et par conséquent la synthèse de leur opposition dans le troisième principe (c.-à-d. la limitation du moi et du non-moi dans le moi) ne fournit qu'une identité relative et partielle. Aucun des trois principes n'est en luimême absolu, et le système fichtéen ne peut sortir de la différenciation du moi et du non-moi et de leur relation réciproque qui définit la conscience réflexive (p. 150-179).

La critique hégélienne du transcendantalisme fichtéen, conçu comme subjectivisme dogmatique, procède en fait du primat que Hegel accorde à l'intérêt de la raison pour l'identité à elle-même, sur lequel se fonde une continuité entre vie et philosophie: le système philosophique, en tant qu'auto-exposition de l'absolu dans sa manifestation rationnelle, satisfait le besoin vital d'identité. La liberté n'est en ce sens qu'un attribut de l'identité: «je suis libre quand je suis en moi-même». Chez Fichte, au contraire, la discontinuité principielle entre philosophie et vie était fondée sur le primat pratique de la liberté dont est dérivé le devoir-devenir-identique-à-soi du moi (p. 164, 165). La critique hégélienne de Fichte présuppose ce primat théorique qui conçoit la philosophie comme auto-construction de l'absolu procédant de l'identité à la totalité. Loin d'être une critique immanente du point de vue fichtéen, elle est une critique intégrative qui réduit celui-ci à un moment du système hégélien (p. 180).

La liberté pratique est ainsi pour Hegel un moment de la liberté absolue qui est réalisée dans le système philosophique par la pensée ou l'idée absolue, dans et pour laquelle pensée et objet s'identifient pour la pensée. Cette liberté est absolue en ce que la pensée est "auprès de soi dans l'autre" (im Anderen bei sich), est négation du négatif, se reconnaît universelle dans et à travers la particularité du pensé. Cette liberté absolue se réalise dans la genèse du système encyclopédique de la philosophie par lequel l'identité absolue se construit comme totalité conceptuelle, comme esprit absolu qui abolit toute extériorité à soi : "L'absolu est l'esprit; telle est la définition suprême de l'absolu » (cité p. 191). Ainsi, la Logique, où le concept s'affirme en tant qu'identité immédiate du sujet et de l'objet, se relativise d'abord par son opposition ou son extériorité aux domaines concrets de la philosophie réelle (Realphilosophie), c.-à-d. la philosophie de la nature et la philosophie de l'esprit qui exposent respectivement une identité objective et subjective du sujet et de l'objet. Le concept ne gagne alors son contenu concret qu'au cours du développement de la philosophie réelle. C'est à l'issue de la dialectique de la philosophie de l'esprit que la pensée auto-consciente se révèle à elle-même, à travers cette genèse conceptuelle de la réalité concrète, comme unité de l'idée logique et de la nature, comme esprit absolu. La liberté pratique, qui domine encore dans les domaines subjectif et objectif de la philosophie de l'esprit, est le dernier niveau avant l'accomplissement encyclopédique de la liberté absolue (p. 181-193).

La reconstruction interne des systèmes de la liberté pratique chez Fichte et Hegel a pu montrer que ces deux systèmes ne peuvent se confronter directement l'un à l'autre ni par une critique immanente (qui part des mêmes présupposés que la position 


\section{Philosophiques / Printemps 2009}

critiquée) ni par une simple critique externe (qui ignore les présupposés de la position critiquée), mais par une critique intégrative par laquelle un système critique les présupposés principiels d'un autre système en les intégrant dans ses propres présupposés (et donc en leur déniant le statut de principe). L'enjeu de la partie conclusive du livre de C. Binkelmann est alors de construire « un point de vue neutre » qui permette de constituer le corrélat positif de la critique du caractère unilatéral de ces deux versions du système de la liberté pratique. La différence fondamentale entre Hegel et Fichte gagne à être éclairée par la question de savoir si la liberté pratique peut être reconduite à un fondement absolu ou pas; cette différence est exprimée dans l'alternative entre les concepts d'origine ou de genèse de la liberté pratique (p. 331-346).

La confrontation directe permet de mieux éclairer les unilatéralités respectives des deux versions ou perspectives du système de la liberté pratique. Chez Fichte, l'absence de fondement et l'extra-mondanité (Grund-und Weltlosigkeit) de la liberté pratique en tant qu'origine lui donnent une position critique pour transformer la réalité, mais elles mettent le moi dans une situation de conscience contradictoire: dans son effort de réalisation infinie de la liberté dans le monde, le moi veut d'une part dépasser le non-moi et tend à le poser dans son unité, mais il doit d'autre part vouloir préserver sa séparation absolue d'avec le non-moi et la réalité objective comme condition de l'auto-affirmation de son origine absolue, de la discontinuité de son auto-position qui serait détruite si le moi devenait le fondement réel de la réalité tout entière (et non plus seulement le fondement idéel de son sens (Sinnstiftung), en tant qu'effort). Au contraire, Hegel ne réduit pas les conditions objectives (de la nature et de l'histoire) à de simples limites à dépasser, mais leur accorde un sens positif dans le procès de réalisation d'une liberté qui retrouve sa mondanité (Welthaftigkeit) et sa réalité objective. Cependant, en reconduisant la liberté subjective à son fondement pour la comprendre comme moment de l'auto-manifestation de l'absolu, Hegel relativise la conscience pratique de la liberté et la nie dans son absoluité irréductible, en tant que croyance infondée (p. 342-351).

Pour donner une issue positive à cette alternance permanente de perspectives entre Fichte et Hegel, l'A. finit par indiquer la voie d'une position intermédiaire pour une théorie actuelle de la liberté pratique en s'appuyant sur l'anthropologie philosophique de Helmuth Plessner. Ce dernier livre les bases d'une synthèse possible entre origine et genèse par sa doctrine de "l'origine devenue» (gewordener Ursprung) de la liberté humaine. D'une part, l'humanité s'inscrit dans le devenir d'un développement continu à la fois organico-naturel et historico-culturel qui mène à l'autocompréhension rationnelle du sujet humain comme liberté, telle qu'elle est advenue dans le cercle culturel européen. D'autre part, c'est la liberté pratique qui donne à l'homme ce pouvoir de donation de sens originaire lui permettant de se poser dans cette genèse continue; c'est donc en se relativisant par son auto-compréhension naturelle et historique que la liberté pratique peut se préserver en tant que "position excentrique» et origine discontinue permanente par rapport à son devenir objectif. Une théorie actuelle de la liberté pratique rend alors d'autant mieux compte du caractère dynamique et subversif de la liberté vivante qu'en la rendant transparente à elle-même par sa genèse philosophique elle fait apparaître son accomplissement comme toujours inachevé dans sa manifestation, toujours ouvert à une nouvelle origine (p. 351-357). 